\title{
Consensus paper on the use of BIVA (Bioelectrical Impendance Vector Analysis) in medicine for the management of body hydration
}

\author{
S. Di Somma ${ }^{\circ}$, H.C. Lukaski, M. Codognotto ${ }^{\S}$, W.F. Peacock, F. Fiorini**, N. Aspromonte ${ }^{\wedge}$, C. Ronco ${ }^{\S}$, S. Santarelli, \\ I. Lalle ${ }^{\circ}$, A. Autunno ${ }^{\circ}$ A. Piccoli ${ }^{\S}$ \\ ${ }^{\circ}$ Department of Emergency Medicine, Sapienza University, Post Graduate School in Emergency-Urgency Medicine, Sant'Andrea Hospital, \\ Rome, Italy \\ "University of North Dakota School of Medicine and Biomedical Sciences Grand Forks, ND, USA \\ ${ }^{\S}$ Department of Medicine, University of Padova, Italy \\ ${ }^{\wedge}$ Department of Emergency Medicine, Cleveland Clinic, Cleveland, OH, USA \\ ** Department of Nephrology, Dialysis and Dietology, SM della Misericordia Hospital, Rovigo, Italy \\ ${ }^{\wedge}$ Cardiovascular Department, San Filippo Neri Hospital, Rome, Italy

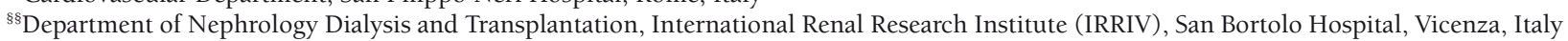 \\ GREAT: Global Research in Acute Conditions Team Network
}

\begin{abstract}
Hydration status assessment remains a challenge overall in subclinical hyper/dehydration status. Biompedance Vector Analysis (BIVA) is a non invasive technique to estimate body mass and water composition by bioelectrical impedance measurements, resistance and reactance. The BIVA method is a unique non invasive technique that allows the combined evaluation of hydration and mass of soft tissues in any clinical condition without knowledge of body weight. This tool can aid physicians to make a fast and correct assessment in facilitating the management of hydration status in critical ill patients in different medicine settings (Cardiology, Nephrology, Gastroenterology, Internal Medicine, Geriatrics and Emergency Medicine).

In Nephrology BIVA contribute to the appropriate diuretic prescriptions in chronic kidney diseases and to the identification of the adequate volume of fluid removed during hemodialysis (reducing hypotension and cramp episode, preventing nocturnal pulmonary edema, ameliorating blood pressure control and maintaining an effective diuresis for long time).

In heart failure patients the combined use of BIVA, biomarkers and
\end{abstract}

\section{Introduction}

Water constitutes approximately $60 \%$ of body weight, varying somewhat with age, sex and amount of body fat. Total body water content (TBW) is distributed between the intracellular fluid (ICF) and the extracellular fluid (ECF) compartments. The ECF is further divided into intravascular and interstitial spaces in a ratio of $1: 4^{1,2}$.

Water homeostasis results from the balance between total water intake and the combined water loss from:

- renal excretion;

- respiratory system;

- skin;

- gastrointestinal sources.

Disorders of body water content are among the most commonly encountered problems in the practices of clinical medicine and in particular of all acute settings. This is in large part due to the fact that many different diseases can potentially disrupt the finely balanced mechanisms that control intake and output of water and solute ${ }^{3}$.

At the same time hydration status assessment remains a challenge. For example severe dehydration can develop with minimal clinical signs and there are not validated laboratory methods to estimate total body water. To estimate acute changes in TBW in real time, bedside ultrasonography allows to: a faster and more accurate diagnosis, distinguish cardiogenic and non cardiogenic dyspnea, support decisions about diuretic therapy, an accurate risk stratification of patients. Ultrafiltration is increasingly recognized as a crucial area of patients with advanced heart failure and fluid overload. The key management goal is careful monitoring of fluid status with early treatment and application of a systematic approach to managing patient during UF. The role of combined technology in assessing hydration has to be defined, but BIVA seems to be of potential value for detecting adequately changes in hydration.

In critical patients arriving at ED, earlier treatment is associated with decreased mortality. BIVA represents a new technology with the potential to objectively define congestive status in ED patients with AHF. Further, it may have utility in identifying patients who require volume overload reduction therapy, as well as serving as an aid to ED disposition.

Future studies aimed to better elucidate the role of BIVA in the management of dehydrated patients should be performed to standardize the use of this technique in these critical patients.

the tools most widely used by physicians are laboratory findings (serum creatinine, electrolytes, urinary and plasmatic osmolarity, haematocrit), chest x-ray, echocardiography and more recently biomarkers (natriuretic peptides) ${ }^{3}$ and bed-side ultrasound. The bioelectrical impedance vector analysis (BIVA) is a non invasive technique to estimate body mass and water composition by bioelectrical impedance measurements, Resistance (R) and Reactance (Xc). BIVA evaluates quickly the body mass in conditions of "normality", extreme obesity, and malnutrition status, and assesses the hydration state of normal (72.7\%-74.3\%), hyperhydration and dehydration ${ }^{4}$.

\section{Principles of bioimpedance analysis}

Use of tetrapolar bioimpedance analysis (BIA) in medical research has expanded from estimation of body composition in healthy individuals to assessment of fluid volume and distribution of individuals with various maladies. Despite the burgeoning application of this method, a general lack of understanding of the fundamental principles and limitations of BIA persists. Impedance is a measure of the opposition to the flow of sinusoidal alternating current in a living organism. Resistance to the applied current occurs in the extracellular and intracellular compartments (Figure 1). 


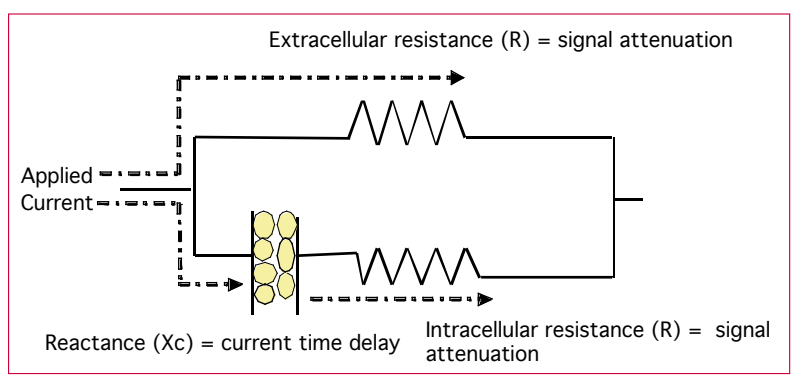

Fig. 1 - A simple electrical circuit representing the body.

Hindrance to the current occurs at the cell membrane and results in reactance or capacitance $e^{5,6}$.

The basic approach is an electro-physical model in which bioconductor volume is inversely related to $\mathrm{R}$ corrected for length or height $\left(\mathrm{V}=\mathrm{L}^{2} / \mathrm{R}\right)^{6,7}$. Resistance, measured at $50 \mathrm{kHz}$ or pre-determined multiple frequencies including $50 \mathrm{kHz}$, body weight, age, and gender were independent variables included in the development of regression models to predict TBW and fat-free mass (FFM). Although this approach resulted in very good correspondence between measured and predicted values in healthy people, the errors in prediction were large (5-10\%) whereas application of these predictions models in clinical groups yielded significant differences ${ }^{8,9}$. Furthermore, analysis of errors revealed wide ranges of error (95\% confidence intervals) in predictions of change in body composition for an individual ${ }^{10}$.

An alternative is the use of bioelectrical impedance spectroscopy or BIS ${ }^{11,12}$. This method uses measurements of $\mathrm{R}$ made sequentially at frequencies of $5 \mathrm{kHz}$ to $2 \mathrm{MHz}$. It extrapolates $\mathrm{R}$ values from low and high frequency to estimate $\mathrm{R}$ of extracellular and intracellular fluids, respectively, with the Cole-Cole model. These values are used to calculate extra- and intracellular resistivities with the Hanai model, which with body weight and height to compute TBW and extracellular water (ECW) and estimate intracellular water (ICW; TBW- ECW). Although theoretically appealing, BIS significantly underestimates TBW in clinical groups and is imprecise for estimates of change in TBW and ECW among individuals ${ }^{13}$, and provides large ranges of error for predictions of individual changes in ICW for individuals ${ }^{14,15}$. Reliance on multiple regression predictions models suffers from various errors. Technical sources include errors of measurement of bioimpedance ( $1 \%$ at $50 \mathrm{kHz}$ ), reference methods including isotope dilution (2-5\%) and the standard error of the estimate generated in the regression model $(5-10 \%)$. These errors propagate and contribute to the imprecision of an estimate for an individual. Electrical and biological errors also contribute as do invalid assumptions of normal and constant hydration of the fat-free body, uniform conduction of the applied current and constant body geometry in cylindrical shape in pathophysiological states ${ }^{16}$

Attention to conditions and circumstances that confound the validity of bioimpedance measures is needed. Electrode placements must ensure adequate contact with skin, shun infusion sites and avoid sweat and emollient accumulation on skin. Presence of high skin temperature, excessive blood loss, severe anemia, excursions of blood electrolytes, and fluid sequestration are pathophysiological disturbances that impact bioimpedance values compared to healthy individuals.

Use of bioimpedance-based regression models to assess body compositional variables is not advised. Problems with propagation of measurement and prediction errors result and violation of basic assumptions of hydration and body geometry in clinical groups contribute to large errors of predictions of compositional variables for individuals. Other approaches are needed to overcome these limitations.

\section{Vector analysis and clinical applications}

In the clinical setting, the evaluation of the patient's hydration status is crucial for subsequent diagnostic steps and treatment. An increase in body fluid volume of 3 to $4 \mathrm{~L}$ is simply detectable as pitting edema. A sub-clinical increase in body fluid volume without edema, and a mild to moderate dehydration are conditions of difficult clinical evaluation ${ }^{17}$. Conventional BIA is based on regression equations ( $\mathrm{R}$ and body weight) that produce estimates of TBW, ECW and ICW. Lean and fat mass are estimated through physiological relationship with soft tissue hydration. Unfortunately, in patients with abnormal hydration, these BIA estimates are biased ${ }^{18}$.

Bioelectrical impedance vector analysis (BIVA) allows a direct assessment of body fluid volume through patterns of vector distribution on the R-Xc plane. Using patterns of the resistancereactance graph (RXc graph) of BIVA it is possible to rank both hydration and mass of soft tissues. Ranking can be performed on repeated measurements of an individual subject or among different subjects. Indeed, the intersubject variability of $\mathrm{Z}$ is represented by the bivariate normal distribution with elliptical probability regions: $50 \%, 75 \%$, and $95 \%$ tolerance ellipses for individual vectors. $\mathrm{R}$ and Xc values are normalized for subjects' height $(\mathrm{H})$ and expressed as $\mathrm{R} / \mathrm{H}$ and $\mathrm{Xc} / \mathrm{H}(\mathrm{Ohm} / \mathrm{m})^{4,19}$.

As with ECG, BIVA uses patterns of impedance vector distribution, which are associated with clinical conditions, without the need of equations or body weight. Changes in tissue hydration status below $500 \mathrm{~mL}$ are detected and ranked. A simple algorithm with few operational rules has been derived for interpreting impedance vector position and migration on the RXc graph at the bedside in any clinical condition ${ }^{20}$.

Vector position on the RXc graph is interpreted following two directions on the R-Xc plane, 1) vector displacements parallel to the major axis of tolerance ellipses indicate progressive changes in tissue hydration, that is dehydration with long vectors, out of the upper pole of the $50 \%$ ellipse, and fluid overload with apparent edema, with short vectors, out of the lower pole of the $50 \%$ ellipse; and 2) peripheral vectors lying in the left side of the major axis, or in the right side of the major axis of tolerance ellipses indicate more or less cell mass, respectively. Fluid removal in malnourished patients is associated with a flat vector migration to the right side, due to an increase in $\mathrm{R}$ without a proportional increase in Xc caused by severe loss of soft tissue mass. This pattern is never observed in vectors lying on the left side of the tolerance ellipses ${ }^{20}$.

The principal use of BIVA is the assessment of hydration status in patients with fluid overload or dehydration which is helpful in prescription of treatments.

In emergency department (ED), BIVA permits to detect dehydration status in subjects admitted for fever, vomiting, diarrhea, perspiration in physical activity and in elderly people living alone. Moreover BIVA was able to detect a "latent peripheral congestion" (vector at the lower pole of the 50\% tolerance ellipse) in 315 patients admitted to the emergency department for acute dyspnea discriminating between cardiac and non cardiac dyspnea (Figure 2). In patients with cardiac dyspnea and latent peripheral congestion the NT pro-BNP concentration was in the "gray zone" 21.

In the critical care setting the estimation of body fluids is neither practical nor reliable. Central venous pressure (CVP) values are used as a guide for fluid infusion: low CVP values are observed with true or relative hypovolemia; conversely, high CVP values indicate true or relative hypervolemia and fluid overload.

BIVA was examined as an indicator of fluid status compared to CVP in 121 ICU patients. Both components of the impedance vector were significantly, linearly, and inversely correlated with 
CVP values. These results support the combined evaluation of tissue hydration by BIVA and CVP in the planning of fluid therapy for ICU patients ${ }^{22}$.

In nephrology, the routine evaluation of hydration status based on body weight and blood pressure changes over time can be misleading because the changes are not uniquely determined by variations in body fluid volume. Use of BIVA can contribute to the appropriate diuretic prescription in chronic kidney disease, to the discrimination between pre-renal acute renal failure due to dehydration respect to other causes of decreased perfusion and to treatment of polyuria following acute tubular necrosis. Moreover BIVA allows the identification of optimal dry weight because it is able to rank changes in tissue hydration status with a minimal detectable change below $500 \mathrm{~mL}$. Adequacy of volume of fluid removed during the hemodialysis and peritoneal dialysis reduces morbidity and improves patient outcomes ${ }^{18,23,24}$. Similar to the hemodialysis cycle, heart failure is characterized by a cyclical fluid overload and removal. The evaluation of optimal "dry" weight determined with BIVA in heart failure patients prevents subclinical dehydration (inappropriate diuretic prescription after disappearance of edema), optimizes ultrafiltration and decreases the rate of re-admission. In initial decompensation of heart, BIVA permits the early detection of fluid accumulation before the appearance of edema ${ }^{25}$ (Figure 3).

Furthermore BIVA enables the identification "by exclusion" volume-dependent hypertension and hypotension. Specifically, a long vector indicating dehydration in a patient with hypertension allows exclusion of volume-dependent hypertension. The same vector in a patient with hypotension is compatible with a volume-dependent hypotension, which can be corrected by reducing ultrafiltration (feedback with vector shortening). A short vector in a patient with hypertension is compatible with volume-dependent hypertension, which can be corrected increasing ultrafiltration (feedback with vector lengthening). The same vector in a patient with hypotension allows exclusion of volume-dependent hypotension ${ }^{26}$. In other internal medicine settings (Gastroenterology, Geriatrics, Long Hospitalization, Nutrition Medicine), the use of BIVA can contribute to the early detection of malnutrition and monitoring parenteral nutrition effects. It prevents sub clinical de-

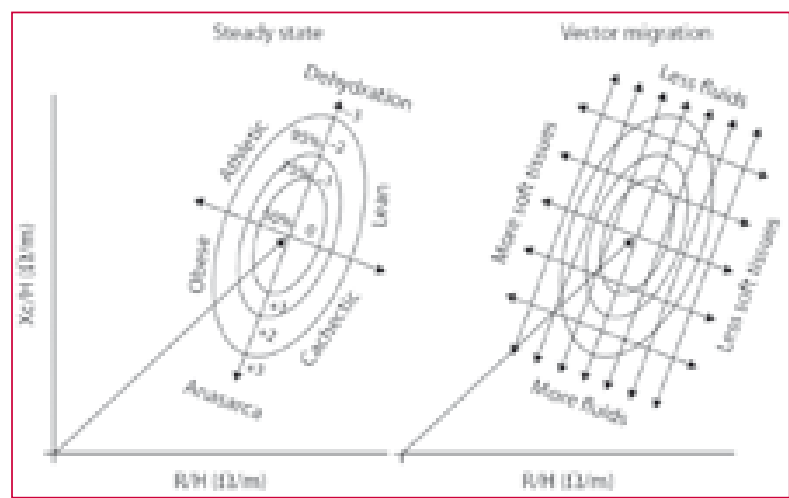

Fig. 2 - Vector displacements parallel to the major axis of tolerance ellipses indicate progressive changes in tissue hydration (dehydration with long vectors, out of the upper pole, and hyperhydration with apparent edema with short vectors, out of the lower pole). Vectors falling (steady state) or migrating (dynamic state) parallel to the minor axis, above (left) or below (right) the major axis of tolerance ellipses indicate more or less cell mass, respectively, contained in soft tissues (i.e. vectors with a comparable $\mathrm{R}$ value and a higher or lower Xc value, respectively). Different trajectories indicate combined changes in both hydration and tissue mass. hydration and fluid accumulation still in the absence of edema and allows the interpretation of body weight change in hypocaloric diet.

\section{BIVA in acute heart failure}

Shortness of breath (SOB) is a frequent ED complaint ${ }^{28,29}$, and is the most common presentation of acute heart failure $(\mathrm{AHF})^{30}$. However, because of many mimics, the diagnosis can be challenging, with 10 to $20 \%$ of initially diagnosed AHF ultimately found to be falsely positive ${ }^{31-33}$. Accurate volume assessment aids diagnostic accuracy, and can guide management ${ }^{34}$. Previous work has demonstrated that delayed therapy in AHF is associated with a $250 \%$ increase in mortality, while the inappropriate use of AHF therapy in non-AHF patients is also associated with an increase in mortality ${ }^{35}$. Despite its importance, current ED volume assessment is little changed in the last 40 years, still predominately relying upon the history, physical and chest $\mathrm{x}$-ray. Bioimpedance vector analysis is an objective and quantitative method of volume assessment. Rapidly and easily performed in the suspected HF patient by the placement of tetrapolar electrode system electrodes at the wrist and ankle, it requires a cooperative supine patient. While being supine may not be possible in severely symptomatic HF patients, it is not this cohort in whom the diagnosis is in question. Rather, it is the more subtle presentation where BIVA has the greatest potential utility.

Volume assessment with BIVA has excellent correlation with the gold standard of deuterium dilution $(r=0.996)^{36}$, but BIVA results are available within 1 minute and at much less expense ${ }^{26}$. BIVA has been successfully used to both diagnose and manage AHF ${ }^{37,25}$. The ED use of BIVA for diagnosis and disposition has been recently reported in a prospective study of 101 patients presenting to the ED with suspected volume abnormalities ${ }^{38}$; of these 31 (30\%) were defined as volume overloaded, of which (25) $80 \%$ were due to AHF. In this analysis, height corrected reactance $(\mathrm{Xc} / \mathrm{H})$ and resistance $(\mathrm{R} / \mathrm{H})$, and calculated bioimpedance $(\mathrm{Z})$ were associated with volume overload (Table 1 ). Reactance was nearly $38 \%$ less in volume overloaded patients, while $\mathrm{R} / \mathrm{H}$ and $\mathrm{Z}$

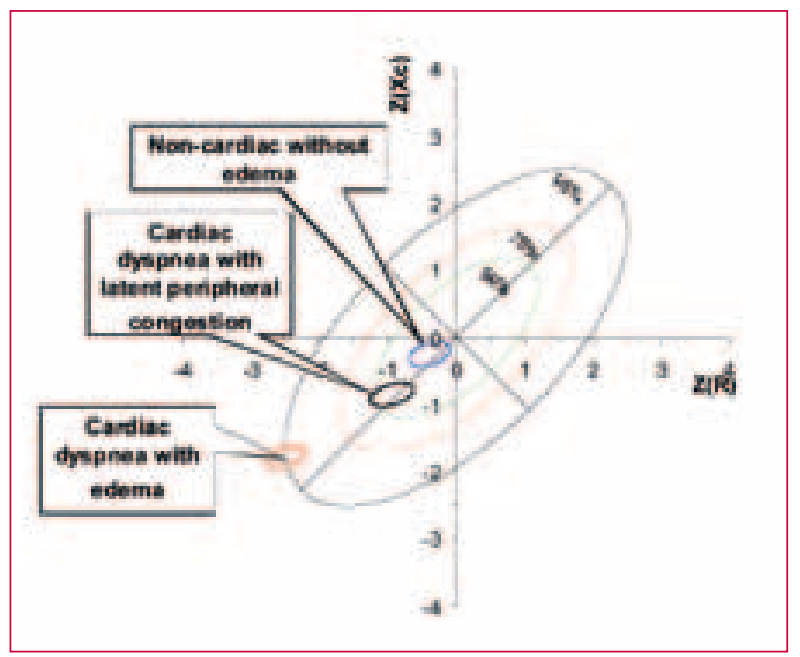

Fig. 3 - The 95\% confidence ellipses of mean vectors are depicted on the bivariate Z-score graph with $50 \%, 75 \%$, and $95 \%$ tolerance ellipses for the individual impedance vector ${ }^{27}$. The group with cardiac dyspnea without edema was close to the lower pole of $50 \%$ tolerance ellipse indicating a mild increase in soft tissue fluid volume (i.e. latent peripheral congestion). The mean vector of patients with edema is shorter, across the lower pole of the $95 \%$ tolerance ellipse. The mean vector of patients with noncardiac SOB is close to the center of tolerance ellipses. 
Table 1

BIVA values predicting volume overload, need for diuretic therapy, and disposition.

\begin{tabular}{|c|c|c|c|c|}
\hline & $\begin{array}{l}\mathrm{Xc} / \mathrm{H}, / \mathrm{m} \\
(95 \% \mathrm{CI})\end{array}$ & $\begin{array}{l}\mathrm{Rz} / \mathrm{H}, / \mathrm{m} \\
(95 \% \mathrm{CI})\end{array}$ & $\begin{array}{l}\mathrm{Z}, / \mathrm{m} \\
(95 \% \mathrm{CI})\end{array}$ & $\begin{array}{l}\text { PA } \\
(95 \% \mathrm{CI})\end{array}$ \\
\hline \multicolumn{5}{|l|}{ Volume overloaded } \\
\hline Volume Overloaded & $\begin{array}{l}18.1 \\
(16.2-19.9)\end{array}$ & $\begin{array}{l}189.3 \\
(172.7-205.9)\end{array}$ & $\begin{array}{l}190.2 \\
(173.6-206.9)\end{array}$ & $\begin{array}{l}5.5 \\
(4.9-6.1)\end{array}$ \\
\hline Non-Volume Overloaded & $29.0(26.9-31.1)$ & $\begin{array}{l}272.9 \\
(256.2-289.6)\end{array}$ & $\begin{array}{l}274.6 \\
(257.8-291.3)\end{array}$ & $\begin{array}{l}6.1 \\
(5.8-6.5)\end{array}$ \\
\hline$P$ value & $<0.0001$ & $<0.0001$ & $<0.0001$ & 0.03 \\
\hline \multicolumn{5}{|l|}{ Loop diuretic need } \\
\hline Loop Diuretic & $\begin{array}{l}18.4 \\
(16.9-20.0)\end{array}$ & $\begin{array}{l}198.2 \\
(183.2-214.0)\end{array}$ & $\begin{array}{l}199.5 \\
(184.1-214.9)\end{array}$ & $\begin{array}{l}5.4 \\
(4.9-5.9)\end{array}$ \\
\hline No Loop Diuretic & $\begin{array}{l}29.8 \\
(27.5-32.0)\end{array}$ & $\begin{array}{l}274.4 \\
(257.2-293.6)\end{array}$ & $\begin{array}{l}277.1 \\
(258.8-295.4)\end{array}$ & $\begin{array}{l}6.2 \\
(5.8-6.6)\end{array}$ \\
\hline P value & $<0.0001$ & $<0.0001$ & $<0.0001$ & 0.007 \\
\hline \multicolumn{5}{|l|}{ ED disposition } \\
\hline Discharge & $\begin{array}{l}28.1 \\
(25.4-30-8)\end{array}$ & $\begin{array}{l}266.9 \\
(247.2-286.7)\end{array}$ & $\begin{array}{l}268.5 \\
(248.7-288.4)\end{array}$ & $\begin{array}{l}6.0 \\
(5.5-6.5)\end{array}$ \\
\hline Admit & $\begin{array}{l}22.8 \\
(20.4-25.1)\end{array}$ & $\begin{array}{l}224.7 \\
(204.0-245.3)\end{array}$ & $\begin{array}{l}225.9 \\
(205.2-246.6)\end{array}$ & $\begin{array}{l}5.9 \\
(5.4-6.3)\end{array}$ \\
\hline$P$ value & 0.002 & 0.001 & 1 & 0.31 \\
\hline
\end{tabular}

were about $30 \%$ less. Further, $\mathrm{Z}$ was the most accurate predictor of volume overload (c statistic $=0.86$, Figure 4 ) when compared to all clinical predictors, including paroxysmal nocturnal dyspnea $(\mathrm{p}<0.0001)$, orthopnea (c-stat $0.63, \mathrm{p}=0.0005)$, rales (c-stat $0.72, p<0.01$ ), jugular venous distension (c-stat $0.66, \mathrm{p}$ $<0.0002$ ), edema (c-stat $0.71, \mathrm{p}<0.01$ ), or the presence of an $\mathrm{S} 3$ or S4 (c-stat $0.53, \mathrm{p}=0.0001)$. Only a BNP $>100 \mathrm{pg} / \mathrm{mL}$ or chest $\mathrm{x}$-ray findings of overload performed equally as well as $\mathrm{Z}$ (BNP c-stat 0.91, p = 0.14, $\mathrm{x}$-ray c-stat $0.76, \mathrm{p}=0.11$ ). There was no correlation between $\mathrm{Z}$ and BNP $(\mathrm{r}=-0.1299, \mathrm{p}=0.28)$. At a

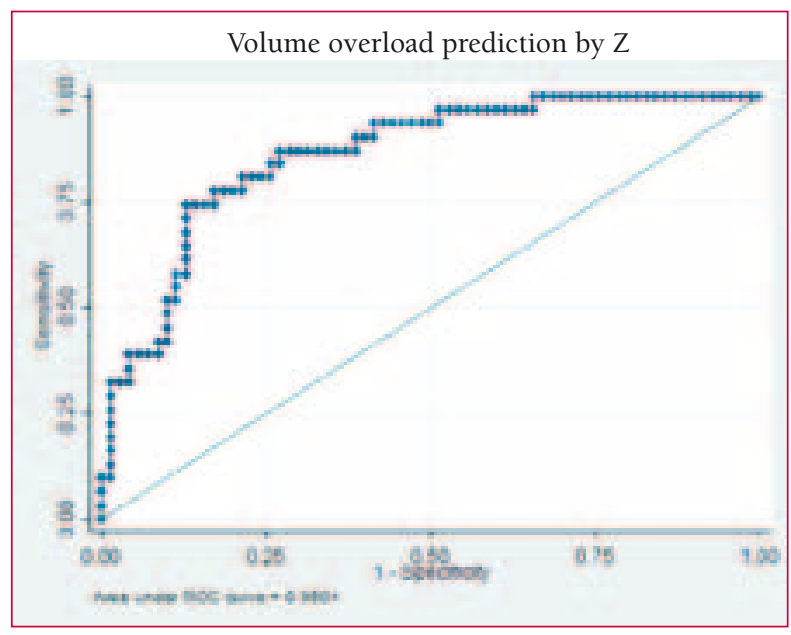

Fig. 4a - Receiver operator characteristic curves for $\mathrm{Z}$ predicting volume overload and the need for volume reduction therapy. cutoff value of $\mathrm{Z} \leq 225 \mathrm{~W} / \mathrm{m}$, BIVA had a sensitivity and specificity of $77 \%$ and $80 \%$ for detecting volume overload, respectively. BIVA measures, see Table 1 , also were valuable for predicting the need for volume reduction therapy (defined as receiving loop diuretics), with superior predictive capability (c-stat 0.83, Figure 4), compared to all clinical parameters and the chest x-ray. Only a BNP $>100 \mathrm{pg} / \mathrm{mL}$ was comparable to BIVA for predicting the need for volume reduction therapy ( $c$-stat $0.82, \mathrm{p}=0.48$ ). Finally, BIVA values were associated with ED disposition and prognosis, see Table 1. Patients discharged home had higher $\mathrm{Xc} / \mathrm{H}(28.1$ vs $22.8 \mathrm{~W} / \mathrm{m}, \mathrm{p}=0.002), \mathrm{R} / \mathrm{H}(266.9$ vs $224.7, \mathrm{~W} / \mathrm{m}$, $\mathrm{p}=0.002)$ and $\mathrm{Z}(268.5$ vs $225.9, \mathrm{~W} / \mathrm{m}, \mathrm{p}=0.001)$. In patients

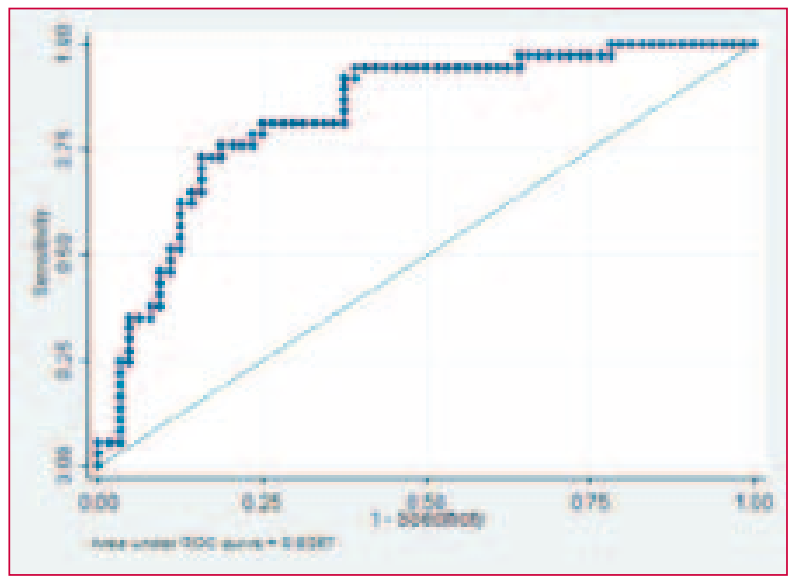

Fig. 4b - Need for diuretic therapy or dialysis assessed by Z. 
hospitalized, $\mathrm{Xc} / \mathrm{H}(\mathrm{r}=-0.36, \mathrm{p}=0.01)$ and PA $(-0.31, \mathrm{p}=0.03)$ had weak correlation with the length of stay in the hospital. Other previous studies have investigated BIVA's ability to help attain dry weight by serial measurements ${ }^{39}$. One study in 94 patients with refractory congestive heart failure, gave high dose furosemide and monitored volume status changes with BIVA at admission and 6 days after. They found that there was a 14\% increase in R/H and a $33 \%$ increase in $\mathrm{Xc} / \mathrm{H}^{40}$. In a pilot study of 186 hospitalized AHF patients, BNP and serial BIVA were used to guide fluid management ${ }^{41}$. This strategy was then validated in 166 AHF patients discharged based on BIVA and BNP targets. They reported lower 6-month readmission rates and cost of care $^{42}$. While further research on BIVA guided therapy is necessary, these early results suggest it may be a useful adjunct for volume management and disposition decisions.

\section{Hemodialysis, hydration, diuresis: the importance of right "dry weight"}

Dialysis is not only a "depurative" therapy, but "dehydrating" technique too. Dialysis patients are unable to maintain water balance and they are variable urine output, ranging from anuria to normal levels. Dialysis therapy is very important to remove excess of fluids intake that accumulate in the body. In dialysis patients, a glomerular filtration rate (GFR) of about $5 \mathrm{~L} /$ day (normal is 140-180 L/day) produces a variable daily urine output ranging from 1.5 to $3.5 \mathrm{~L}$.

Malnutrition is an overt problem in dialysis, correlating with raise in morbidity and mortality and depending of reduction of food intake (inadequate dialysis with anorexia, depression, exaggerate water intake), hyper-catabolism due to dialysis therapy, poor dietetic compliance, poor physical activity and significant drugs intake ${ }^{43}$.

In dialysis, malnutrition and concurrent illness cause protein catabolism with muscle mass reduction and total body water increase induced by artificial preservation of steady body weight through the dialytic procedures. Dialysis patients are unable to modulate total body content (hence body weight) during starvation and refeeding: body weight modification is achieved artificially by dialysis. In hemodialysis patients "exact" body weight (named also "dry" weight) may be defined as post procedural weight at which the patient is as close as possible to a normal hydration state without experiencing symptoms indicative of hyper- or de-hydration at or after the end of hemodialysis treatment. In dialysis patients, asymptomatic variation in body weight $( \pm 5 \mathrm{Kg})$ can derive from overhydration with consequent leg edema, pulmonary edema (and possible acute dialysis), artery hypertension, dehydration with consequent artery hyper/ hypo-tension, asthenia, hypotension, nausea, vomiting during $\mathrm{HD}$, and muscle cramps.

In our department, as of 1997 we have set routinely BIA assessments on 98 consecutive HD patients at two months frequency and/or whenever needed (recent illness, cramps or hypotension onset, etc). We tailor the "dry weight" prescription using BIVA (RXc Graph), completing the former rule tied to body weight or other parameters (HD hypotension, muscle cramps, arterial hypertension).

As a results of this new approach of "dry weight" control in 98 consecutive HD patients, we noted a reduction of intra-HD hypotension episodes ( 20 vs $8 \%$ ), reduction of intra-HD cramps episodes ( 27 vs 14\%), reduction of acute additional (nocturnal or not) HD in chronic HD patients (4\% vs none), persistent diuresis (> $1 \mathrm{~L}$ /day) after $18 \mathrm{HD}$ month.

The causes of urine outfalls in dialysis patients are reduced volume expansion, reduced high urea load per nephron, activation of nephrotoxic inflammatory mediators during HD or PD, higher diastolic blood pressure, increased proteinuria, hypotension with

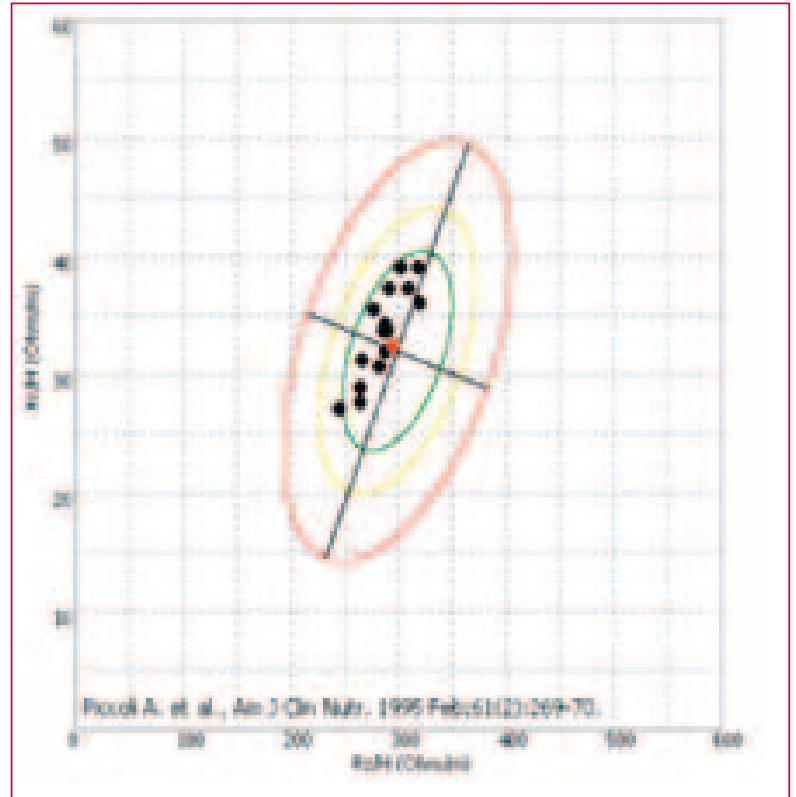

Fig. 5 - Distribution of subjects with residual diuresis.

$\mathrm{HD}$, dehydration episodes with $\mathrm{PD}$, exposure to nephro-toxic drugs. In our patients the lack of dehydration assessed with BIVA permit a long term conservation of consistent diuresis (Figure 5). The HD patients with residual but consistent diuresis, have some advantages: greater water intake, greater nutritional intake/status, significant contribution to the removal of potential uremic toxins, better psycologic condition.

In HD patients BIA obtains two different goals, body water evaluation, and morbidity and mortality predictions. Therefore, BIA can be considered as a part of the clinical examination for the evaluation of both hydration and nutritional status. These properties seem to reduce hemodialysis hypotension and cramp episodes, to prevent nocturnal pulmonary edema, to ameliorate blood pressure control and to maintain an effective diuresis for long time.

\section{BIVA in ultrafiltration}

Traditional diuretic therapy for patients with acute decompensated heart failure (ADHF) can often be ineffective due to patients developing diuretic resistance. In fact, according to the Acute Decompensated Heart Failure National registry (ADHERE), the primary reason for ADHF hospitalization is congestion in patients refractory to oral diuretics ${ }^{44}$. In these patients fluid removal by ultrafiltration (UF), an innovative, non-drug-based option, represents a significant advance in management of congestion. Compared to diuretic therapy, UF can remove the excess of salt and water from the blood stream with faster resolution of systemic and/or pulmonary congestion and recovery of sensitivity to diuretics while avoiding effects on kidney function, heart rate, blood pressure, and electrolytes ${ }^{45}$. Early UF in ADHF patients can not only improve rehospitalization rates but also safely and effectively reduce congestion in these patients ${ }^{46}$. Although much emphasis has been placed on UF management, a crucial area is the monitoring of hydration and fluid balance problems such as how much fluid should be removed and when an optimal fluid state has been achieved for a specific patient. Maintenance of blood volume, vascular tone, and hemodynamic stability depends on a set of interactions between the heart and kidney during $\mathrm{UF}^{47}$.

This therapy has many theoretical advantages because a con- 
stant slow rate of UF allows time for vascular refilling and effective control of fluid balance while maintaining hemodynamic stability (Figure 6). Inaccurate information about fluid distribution can change drug disposition and cause serious acute and long-term complications for a UF patients. So, to optimize fluidrelated outcomes, reliable and prompt detection of abnormalities in fluid balance are essential to enable the appropriate therapeutic changes during UF. In view of this goal, more sensitive and objective measures of hydration are needed.

Knowledge of patient fluid distribution by BIVA would be very useful for guiding UF therapy as well as nutritional support. A dinamic measurement of ECW is the prerequisite for bioimpedance monitoring during UF that accounts for changes in regional fluid distribution when a considerable amount of fluid is removed from the body without adverse effects. Extracellular and intracellular volume expansion can be quantified using a variety of bioimpedance nomogram. Titration of fluid removal to such measurements might ensure avoidance of excessive fluid removal. For UF, blood volume monitoring by a continuous hematocrit measurement enables the early detection of inadequate intravascular refilling, which might enable fluid removal rates to be adjusted. However, hypovolemia and renal hypoperfusion can occur in patients with ADHF if excessive fluid removal is pursued with extracorporeal therapy. Thus, accurate assessment of fluid status and careful definition of targets are needed ${ }^{-}$ Impedance methods are increasingly being used to fulfill this gap of knowledge used stand alone or in conjunction with other techniques such as ultrasound measurement of inferior vena cava (IVC) diameter, lung ultrasound (LUS) very useful in the evaluation of the extravascular lung water and measurement of serum natriuretic peptide concentrations.

Ultrasound can measure IVC diameter and "collapsibility" with respiration, which have been shown to reflect the hydration state in critically ill and cardiorenal patients ${ }^{48}$.

Finally, brain natriuretic peptide (BNP) and related molecules, which are biomarkers of congestive cardiac failure, have been correlated with echocardiographic and bioimpedance measures of fluid overload in patients with $\mathrm{HF}^{49}$. Measurement of BNP could aid assessment of the degree of fluid overload, particularly in the presence of coexisting cardiac failure; although its interpretation would require a disconnection between the relative contributions of baseline cardiac disease and superimposed fluid overload to the elevated BNP level ${ }^{50}$. Furthermore, blood concentrations are also affected by renal failure, and so they are not at present useful as pure markers of hydration.

Ultrafiltration is increasingly recognized as a crucial area of patients with advanced heart failure and fluid overload. The key management goal is careful monitoring of fluid status with early treatment and application of a systematic approach to managing

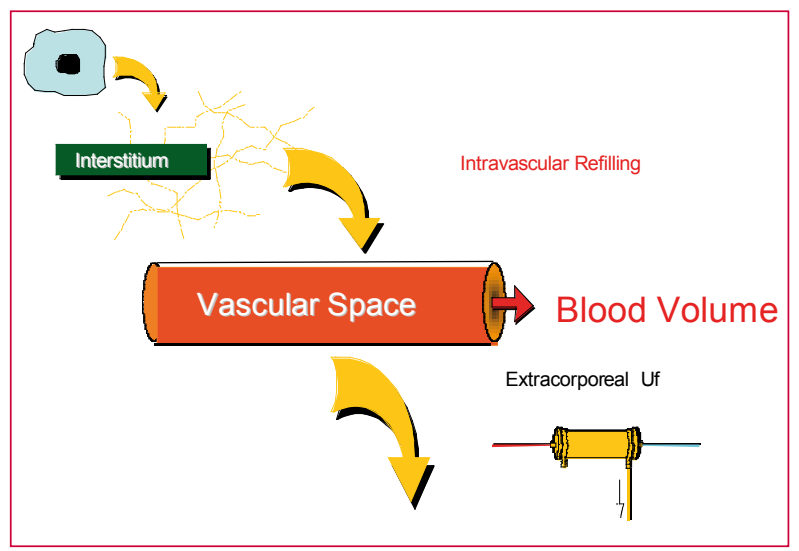

Fig. 6 - Mechanism of ultrafiltration patient during UF. The role of combined technology in assessing hydration has to be defined, but BIVA seems to be of potential value for detecting adequately changes in hydration.

\section{BIVA for management of body hydration in Emergency room}

A correct evaluation of hydration state or water homeostasis is an important component in the management of critically ill patients in the ED.

Although clinical assessment remains the mainstay of estimating hydration state in patients, it could be challenging in all acute diseases to evaluate subtle hyper or dehydration, which may result in increased short and long-term morbidity. However, hydration status assessment is not easy to evaluate because of the inaccuracy of clinical evaluation and lack of techniques ${ }^{51}$. For instance, in chronic heart failure patients, edema is not usually detectable until the interstitial fluid volume has risen to about $30 \%$ above normal ${ }^{52}$; on the other hand, severe dehydration can develop with minimal clinical signs. Also the efficacy of any assessment marker for detecting disturbances in homeostasis depends critically on the nature of body fluids losses and challenges exist when patients present with many simultaneous co-morbidities and confounders ${ }^{53}$.

For these reasons a single gold standard for hydration assessment is yet not possible. In patients with a high severity of illness earlier treatment is associated with decreased mortality and patient's outcome depends on a rapid and accurate volume assessment ${ }^{53}$. Consequently the main purpose of ED physicians is to immediately distinguish between normal hydrated, dehydrated and hyperhydrated patients ${ }^{3}$.

The conventional tools that ED physician can use to assess hydration status are:

Medical history: dyspnea on exertion, edema, paroxysmal nocturnal dyspnea, orthopnea in heart failure (HF); vomiting, diarrhea, decreased oral intake in dehydration status.

Physical examination: lung sounds (rales), peripheral edema, jugular venous distention, hepatojugular reflux (in $\mathrm{HF}$ ); tongue dryness, longitudinal tongue furrows, dryness of mucous, confusion, speech difficulty, sunken eyes, later orthostatic symptoms, hypotension, third heart sound (in dehydrated patients).

Laboratory: Natriuretic Peptides in HF; plasma osmolarity provides the best potential measure for static hydration assessment, while dynamic hydration assessment is best described by using Plasma osmolarity, Urinary specific gravity and Body mass.

Instrumental:

1. Chest radiography: dilated upper lobe vessels, cardiomegaly, interstitial edema, enlarged pulmonary artery, pleural effusion, alveolar edema, prominent superior vena cava, Kerley lines ( $20 \%$ of HF patients have an initial negative chest radiography). Chest radiography is not particularly helpfull to detect hypovolemia, but can determine the nature of patients symptoms.

2. Ecocardiography: heart dimensions, ventricular function, ejection fraction, left atrial and pulmonary artery capillary wedge pressure, miocardial filling, ejection fraction.

3. Thoracic ultrasonography: B-lines (alveolar, interstitial syndrome), pleural effusion

4. Vena Cava Index assessment: inferior vena cava diameter and collapse index.

In patients with hyperhydration state due to heart failure, some authors showed as the reactance is strongly related with the BNP values and the NYHA functional classes ${ }^{54}$. Other authors found in critically ill patients a correlation between impedance and central venous pressure ${ }^{22}$. 


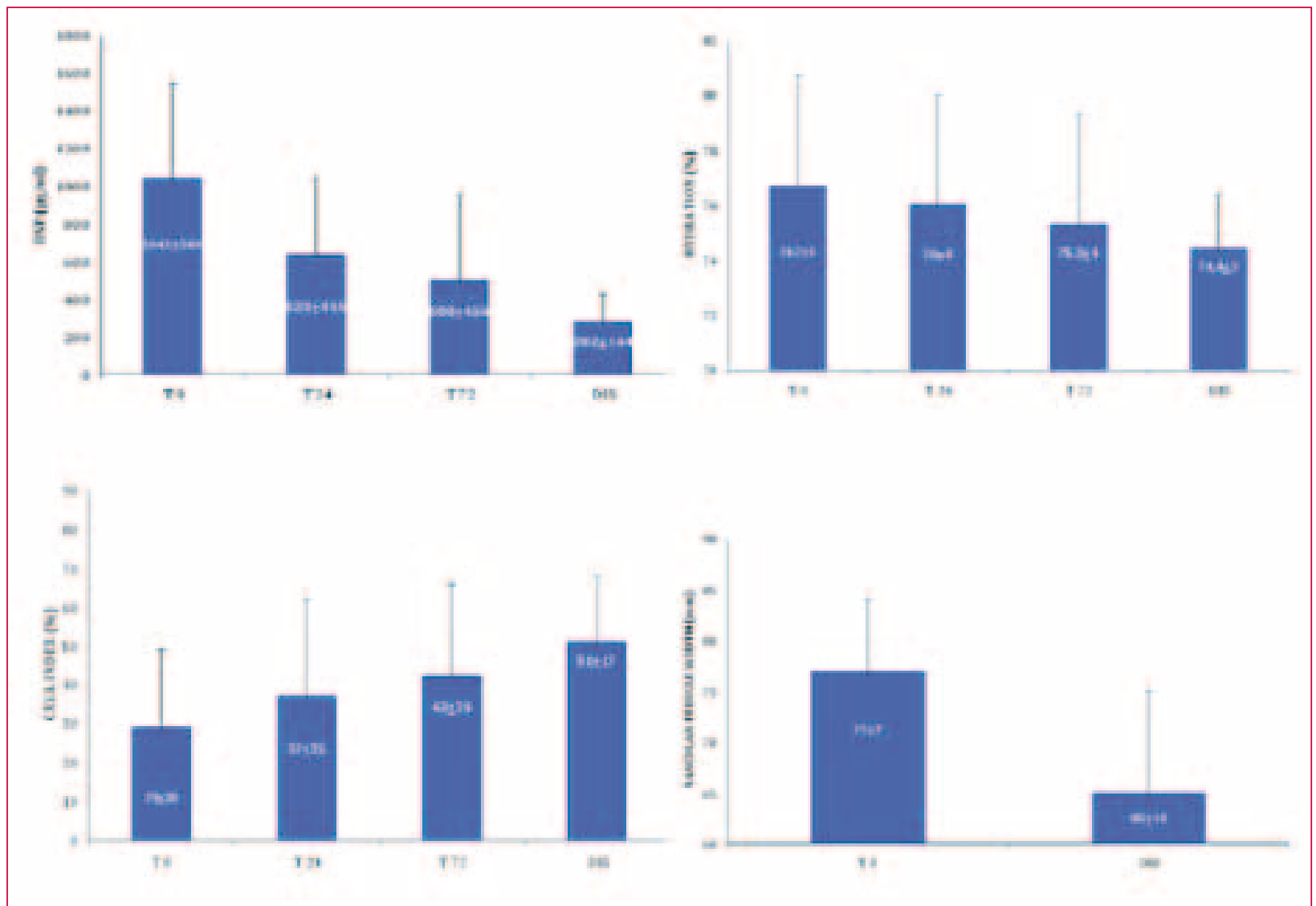

Fig. 7 - Effect of diuretic treatment in patients with acute decompensated heart failure: monitoring brain natriuretic peptide (BNP) and fluid content (Hydration). Hydration state was analyzed at admission to the emergency department, 24 and 72 hours after admission, and at discharge, together with BNP and ultrasonographic measurement of the caval index and vascular pedicle width obtained through chest radiography. All the methods confirmed a good response to diuretic therapy with the shift of fluids from interstitial spaces.

For the ED physicians, BIVA could represent the ideal hydration assessment technique because it measures fluids volume and concentration in real time, it has excellent precision, accuracy and reliability, it is portable, not invasive, inexpensive, safe and simple to use. Moreover, the combined use of both BIVA and BNP improves the management of heart failure patients because it is useful to reach a faster and more accurate diagnosis, it is a valid support to distinguish cardiogenic dyspnea from noncardiogenic dyspnea and supports decisions about diuretic therapy. Furthermore the combination of BIVA and BNP can identifies patients with higher probability of events (death or rehospitalization) $)^{55}$ and allows a more accurate risk stratification of patients undergoing diuretic therapy ${ }^{25}$.

NGAL is a valid biomarker that can ameliorate hydration status evaluation and infusion therapy management both in congestive and in dehydrated patients, by monitoring renal function.

Thoracic ultrasonography is another valid tool to assess hydration status. More important is the possibility to have a "bed side ultrasonography", with newer portable scanners that ED physician can use at the bedside to detect alveolar-interstitial syndrome or ultrasound pattern of diffuse and multiple comet tail artifacts in diseases involving lung interstitium. In heart failure patients it shows a better accuracy than radiography, especially in the detection of small amount of pleural effusion. Exploiting the less dynamic diameter of the inferior vena cava (IVC) dilated by the venous congestion, it is possible to assess a volume overload state (respiratory variation of IVC diameter it has been found to achieve a $92 \%$ sensitivity and $84 \%$ specificity for the diagnosis of HF) (Figure 7). IVC diameter and its collapse index allow to detect a dehydration status. While ultrasonography, through vena cava index evaluation, estimates intravascular fluids volume, BIVA allows measure of intra and extra cellular water.

The introduction of BIVA technique in our clinical experience showed positive results. In our ED, we evaluated hydration status at the admission, 24, 72 hours after admission and at the discharge time in acute heart failure patients using a multidimentional approach: together with BIVA, we evaluated BNP, ultrasonographic measurement of the Caval Index and vascular pedicle width obtained through chest radiography.

From our data it is possible to conclude that the BIVA measurements are strictly related with the other three methods. In our experience BIVA and Caval Index showed a significant and indirectly proportional correlation at the time of ED presentation and 24 and 72 hours after hospital admission ${ }^{55}$. Moreover at admission we found an inverse relationship between BIVA and reduced urine output.

Our results confirm the strong correlation between hyperhydration and central venous congestion and between hyperhydration and oliguria. The correlation between BIVA and diuresis at the time 72 hours became directly proportional. This confirms the good response to diuretic therapy with the shift of fluids from interstitial spaces: the more the patients were hyperhydrated the more the diuresis was conspicuous. BIVA confirmed the utility to evaluate the coming back to a normal hydration state at the time $24 \mathrm{~h}$ and $72 \mathrm{~h}$ with a specificity of $100 \%$ (AUC 0.7, p $=0.04,24 \mathrm{~h}$; AUC 0.9, p = 0.0001, $72 \mathrm{~h}$ ). The efficacy of diure- 
tic therapy and the validity of BIVA measurements were besides confirmed by normalization of BNP, vascular pedicle width and Caval Index values at discharge (Figure 7). These results suggest that BIVA, more than clinical empirical signs, can be useful to manage the diuretic therapy in critical heart failure patients in ED. Another interesting application of BIVA is the evaluation of the hydration state and body mass in cardiac cachexia patients in which weight loss due to body wasting is particularly dangerous. In fact cardiac cachexia is a terminal multi-factorial stage of heart failure resulting in catabolic imbalance on the body leading to the loss of lean and fat mass. This condition, when present, can confound the clinical evaluation. In these patients BIVA would be useful in assessment of correct hydration but also of body mass loss. In the future, the reliability of BIVA in cardiac cachexia patients should be validated ${ }^{3}$.

\section{Conclusions}

The BIVA method is a unique non invasive technique that allows the combined evaluation of hydration and mass of soft tissues in any clinical condition without knowledge of body weight.

This tool can aid physicians to make a fast and correct assessment in facilitating the management of hydration status in critical ill patients in different medicine settings (Cardiology, Nephrology, Gastroenterology, Internal Medicine, Geriatrics and Emergency Medicine).

BIVA represents a new technology with the potential to objectively define congestive status in ED patients with AHF. Further, it may have utility in identifying patients who require volume overload reduction therapy, as well as serving as an aid to ED disposition.

The combined use of clinical evaluation, BIVA, biomarkers such as natriuretic peptides and renal biomarkers such as NGAL and Cystatin $\mathrm{C}$ and bed side ultrasonography must be considered a multidimensional, non invasive, rapid and accurate approach in the management of hydration status in patients with acute heart failure also as response to specific therapy.

The routine use of BIVA in hemodialysis patients permits to evaluate body water and to modulate body weight resulting from variation on fat free mass or fatty mass due to nutritional and physical activity changes or intercurrent illness.

Ultrafiltration is increasingly recognized as a crucial area of patients with advanced heart failure and fluid overload. The key management goal is careful monitoring of fluid status with early treatment and application of a systematic approach to managing patient during UF. The role of combined technology in assessing hydration has to be defined, but BIVA seems to be of potential value for detecting adequately changes in hydration.

Future studies aimed to better elucidate the role of BIVA in the management of dehydrated patients should be performed to standardize the use of this technique in these critical patients.

\section{Key points}

- Hydration status assessment remains a challenge overall in subclinical hyper/dehydration status.

- BIVA is a non invasive technique to estimate body mass and water composition by bioelectrical impedance measurements, resistance and reactance.

- In Nephrology BIVA contribute to the appropriate diuretic prescriptions in chronic kidney diseases and to the identification of the adequate volume of fluid removed during hemodialysis (reducing hypotension and cramp episode, preventing nocturnal pulmonary edema, ameliorating blood pressure control and maintaining an effective diuresis for long time).

- In heart failure patients the combined use of BIVA, biomarkers and bedside ultrasonography allows to: a faster and more accurate diagnosis, distinguish cardiogenic and non cardiogenic dyspnea, support decisions about diuretic therapy, an accurate risk stratification of patients.

- In ultrafiltration BIVA is very useful for guiding therapy as well as nutritional support.

- In critical patients arriving at ED, earlier treatment is associated with decreased mortality.

- Future studies aimed to better elucidate the role of BIVA in the management of dehydrated patients should be performed to standardize the use of this technique in these critical patients.

\section{References}

1. Verbalis JG. Disorders of body water homeostasis. Best Pract Res Clin Endocrinol Metab 2003; 17(4): 471-503.

2. Brenner \& Rector. Il rene. Verducci Editore, 2002.

3. Di Somma $S$ et al. Fluid Assessment and management in emergency department. Contrib Nephrol 2010; 164: 227-236

4. Piccoli A, Rossi B, Pillon L, Bucciante G. A new method for monitoring body fluid variation by bioimpedance analysis: the RXc graph. Kidney Int 1994; 46(2): 534-539.

5. Lukaski HC. Biological indices considered in the derivation of the bioelectrical impedance analysis. Am J Clin Nutr 1996; 64(Suppl): 397S-404S.

6. Nyboer J. Electrical Impedance Plethysmography: The Electrical Resistive Nature of the Blood Pulse Volume. Charles C. Thomas, Springfield (IL), 1959.

7. Hoffer EC, Meador CK, Simpson DC. Correlation of wholebody impedance with total body water volume. J Appl Physiol 1969; 27: 531-534.

8. Kyle UG, Bosaeus I, De Lorenzo AD et al. Bioelectrical impedance analysis - part 2: utilization in clinical practice. Clin Nutr 2004; 23: 1430-1453.

9. Buchholz AC, Bartok C, Schoeller DA. The validity of bioelectrical impedance models in clinical populations. Nutr Clin Prac 2004; 19: 433-446.

10. Savastano S, Belfiore A, Di Somma C et al. Validity of bioelectrical impedance analysis to estimate body composition changes after bariatric surgery in premenopausal women. Obes Surg 2010; 20: 332-339.

11. Jaffrin MY, Morel H. Body fluid volumes measurements by impedance: a review of bioimpedance spectroscopy (BIS) and bioimpedance analysis (BIA) methods. Med Eng Phys 2008; 30: 1256-1269.

12. Matthei JR. Bioimpedance measurements of human body composition: critical analysis and outlook. Expert Rev Med Devices 2008; 5: 239-261

13. Cox-Reijven PL, van Kreel B, Soeters PB. Bioelectrical impedance measurements in patients with gastrointestinal disease: validation of the spectrum approach and a comparison of different methods for screening for nutritional depletion. Am J Clin Nutr 2003; 78: 1111-1119.

14. Earthman C, Traughber D, Dobratz J, Howell W. Bioimpedance spectroscopy for clinical assessment of fluid distribution and body cell mass. Nutr Clin Prac 2007; 22: 389-405.

15. Mager JR, Sibley SD, Beckman TR et al. Multifrequency bioelectrical impedance analysis and bioelectrical impedance spectroscopy for monitoring fluid and body cell mass changes after gastric bypass surgery. Clin Nutr 2008; 27: 832-841.

16. Foster KR, Lukaski HC. Whole body impedance: what does it measure? Am J Clin Nutr 1996; 64(Suppl): 388S-396S.

17. Guyton AC. Textbook of medical physiology. Saunders, Philadelphia, 1991, p. 282.

18. Piccoli A; The Italian Hemodialysis-Bioelectrical Impedance Analysis (HD-BIA) Study Group. Identification of operational clues to dry weight prescription in hemodialysis using bioimpedance vector analysis. Kidney Int 1998; 53(4): 1036-1043. 
19. Piccoli A, Nigrelli S, Caberlotto A et al. Bivariate normal values of the bioelectrical impedance vector in adult and elderly populations. Am J Clin Nutr 1995; 61(2): 269-270.

20. Piccoli A. Patterns of bioelectrical impedance vector analysis: learning from electrocardiography and forgetting electric circuit models. Nutrition 2002; 18(6): 520-521.

21. Piccoli A, Codognotto M, Cianci V et al. Differentiation of cardiac and non cardiac dyspnea using bioelectrical impedance vector analysis (BIVA). J Card Fail in press.

22. Piccoli A, Pittoni G, Facco E et al. Relationship between central venous pressure and bioimpedance vector analysis in critically ill patients. Crit Care Med 2000; 28(1): 132-137.

23. Piccoli A; Italian CAPD-BIA Study Group. Bioelectric impedance vector distribution in peritoneal dialysis patients with different hydration status. Kidney Int 2004; 65(3):1050-1063.

24. Pillon L, Piccoli A, Lowrie EG et al. Vector length as a proxy for the adequacy of ultrafiltration in hemodialysis. Kidney Int 2004; 66(3): 1266-1271.

25. Valle R, Aspromonte N, Milani L et al. Optimizing fluid management in patients with acute decompensated heart failure (ADHF): the emerging role of combined measurement of body hydration status and brain natriuretic peptide (BNP) levels. Heart Fail Rev 2011; 16 (6): 519-529.

26. Piccoli A. Whole body- single frequency bioimpedance. Contrib Nephrol 2005; 149: 150-161.

27. Piccoli A, Pillon L, Dumpler F. Impedance vector distribution by sex, race, body mass index, and age in the United States: standard reference intervals as bivariate $\mathrm{Z}$ scores. $\mathrm{Nu}$ trition 2002; 18(2): 153-167.

28. Pitts SR, Niska RW, Xu J et al. National Hospital Ambulatory Medical Care Survey: 2006 emergency department summary. Natl Health Stat Report 2008; 7: 1-38.

29. Schappert SM, Rechtsteiner EA. Ambulatory medical care utilization estimates for 2007. Vital Health Stat 2011; 169: 1-38

30. Goldberg RJ, Spencer FA, Szklo-Coxe M et al. Symptom presentation in patients hospitalized with acute heart failure. Clin Cardiol 2010; 33(6): E73-E80

31. Mard S, Nielsen FE. Positive predictive value and impact of misdiagnosis of a heart failure diagnosis in administrative registers among patients admitted to a University Hospital cardiac care unit. Clin Epidemiol 2010; 2: 235-239.

32. Dao Q, Krishnaswamy P, Kazanegra R, et al. Utility of B-type natriuretic peptide in the diagnosis of congestive heart failure in an urgent-care setting. J Am Coll Cardiol 2001; 37(2): 379-385

33. Collins SP, Lindsell CJ, Peacock WF, et al. Clinical characteristics of emergency department heart failure patients initially diagnosed as non-heart failure. BMC Emerg Med 2006; 6: 11.

34. Jessup M, Abraham WT, Casey DE, et al. 2009 Focused Update: ACCF/AHA Guidelines for the Diagnosis and Management of Heart Failure in Adults: A Report of the American College of Cardiology Foundation/American Heart Association Task Force on Practice Guidelines Developed in Collaboration With the International Society for Heart and Lung Transplantation. J Am Coll Cardiol 2009; 53(15): 1343-1382.

35. Wuerz RC, Meador SA. Effects of prehospital medications on mortality and length of stay in congestive heart failure. Ann Emerg Med 1992; 21(6): 669-764.

36. Kushner R, Schoeller D, Fjeld C et al. Is the impedance index (ht2/R) significant in predicting total body water? Am J Clin Nutr 1992; 56(5): 835-839.

37. Parrinello G, Paterna S, Di Pasquale P et al. The Usefulness of Bioelectrical Impedance Analysis in Differentiating Dyspnea Due to Decompensated Heart Failure. J Card Fail 2008; 14(8): 676-686.

38. Tuy T, Talati A, Fiessinger L et al. Detection of Acute Heart Failure in the Emergency Department with Bioimpedance
Vector Analysis. Ann EM 2011 abstract presented at the American College of Emergency Physician's Scientific Assembly, San Francisco (CA), Oct, 2011

39. Coodley EL, Segal JL, Smith DH et al. Bioelectrical impedance analysis as an assessment of diuresis in congestive heart failure. Ann Pharmacother 1995; 29(11): 1091-1095.

40. Paterna S, Di Pasquale P, Parrinello G et al. Changes in brain natriuretic peptide levels and bioelectrical impedance measurements after treatment with high-dose furosemide and hypertonic saline solution versus high-dose furosemide alone in refractory congestive heart failure: a double-blind study. J Am Coll Cardiol 2005; 45(12): 1997-2003.

41. Valle R, Aspromonte N, Giovinazzo P et al. B-type natriuretic Peptide-guided treatment for predicting outcome in patients hospitalized in sub-intensive care unit with acute heart failure. J Card Fail 2008; 14(3): 219-224.

42. Valle R, Aspromonte N, Carbonieri E et al. Fall in readmission rate for heart failure after implementation of B-type natriuretic peptide testing for discharge decision: a retrospective study. Int J Cardiol 2008; 126(3): 400-406.

43. Locatelli F, Fouque D, Heimburger O et al. Nutritional status in dialysis patients: a European consensus. Nephrol Dial Transplant 2002; 17: 563-572.

44. Adams KF Jr, Fonarow GC, Emerman CL et al.; ADHERE Scientific Advisory Committee and Investigators. Characteristics and outcomes of patients hospitalized for heart failure in the United States: rationale, design, and preliminary observations from the first 100,000 cases in the Acute Decompensated Heart Failure National Registry (ADHERE). Am Heart J 2005; 149: 209-216.

45. Ronco C., Bellomo R, Ricci, Z. Hemodynamic response to fluid withdrawal in overhydrated patients treated with intermittent ultrafiltration and slow continuous ultrafiltration: role of blood volume monitoring. Cardiology 2001; 96: 196-201.

46. Costanzo MR, Guglin ME, Saltzberg MT et al. Ultrafiltration versus intravenous diuretics for patients hospitalized for acute decompensated heart failure. J Am Coll Cardiol 2007; 49: 675-683.

47. Clark WR, Paganini E, Weinstein D et al. Extracorporeal ultrafiltration for acute exacerbation of chronic heart failure: report from the acute dialysis quality initiative. Int J Artif Organs 2005; 8: 466-476.

48. Ng Kam Chuen MJ, Lip GY, Macfadyen RJ. Performing repeated noninvasive bedside measures of volume response to intravenous furosemide in acute pulmonary edema: a feasibility assessment. Cardiovasc Ther 2009; 27(2): 89-95.

49. Parrinello G, Paterna S, Di Pasquale P et al. The usefulness of bioelectrical impedance analysis in differentiating dyspnea due to decompensated heart failure. J Card Fail 2008; 14: 676-686.

50. Valle R, Aspromonte N. Use of Brain Natriuretic Peptide and Bioimpedance to Guide Therapy in Heart Failure Patients. Contrib Nephrol 2010; 164: 209-216.

51. Armstrong LE. Assessing hydration status: the elusive gold standard. J Am Coll Nutr 2007; 26(5 suppl): 5755-5845.

52. Bozzetto S, Piccoli A, Montini G. Bioelectrical impedance vector analysis to evaluate relative hydration status. Pediatr Nephrol 2010; 25: 229-334.

53. Peacock WF, Soto KM. Current technique of fluid status assessment. Cong Heart Fail 2010; 16 (suppl 1): S45-S51.

54. Castillo Martínez L, Colín Ramírez E, Orea Tejeda A et al. Bioelectrical impedance and strength measurements in patients with heart failure: comparison with functional class. Nutrition 2007; 23(5): 412-418.

55. Di Somma S., De Berardinis B, Bongiovanni C et al. Use of BNP and bioimpedence to drive therapy in heart failure patients. Cong Heart Fail 2010; 16 (suppl 1): S56-S61. 